\title{
Reprodução de Trichospilus diatraeae em Diatraea saccharalis após três gerações em Tenebrio molitor ${ }^{1}$
}

\author{
Daniele Fabiana Glaeser ${ }^{2}$, Fabricio Fagundes Pereira ${ }^{3}$, \\ Elizangela Leite Vargas ${ }^{2}$, Vanessa Rodrigues Ferreira Calado², Kellen Favero ${ }^{3}$
}

\begin{abstract}
Reproduction of Trichospilus diatraeae in Diatraea saccharalis after three generations in Tenebrio molitor

The successive rearing of parasitoids in factitious hosts may affect its biological quality. Trichospilus diatraeae Cherian \& Margabandhu, 1942 (Hymenoptera: Eulophidae) has been studied for the biological control of sugarcane borer [Diatraea saccharalis (Fabricius, 1794) (Lepidoptera: Crambidae)]. This study aimed to evaluate whether the rearing of $T$. diatraeae for three generations in the factitious host Tenebrio molitor Linnaeus, 1758 (Coleoptera: Tenebrionidae) affects its reproductive performance, when subsequently reared in the natural host pupae $D$. saccharalis. Two groups of $T$. diatraeae were reared separately for three generations: one in pupae of T. molitor and the other in pupae of $D$. saccharalis. Subsequently, 20 pupae of $D$. saccharalis were exposed, for 72 hours, to the parasitism of $T$. diatraeae females reared earlier in pupae of T. molitor or D. saccharalis. The successive rearing of T. diatraeae in the factitious host did not affect the number of pupae parasitized and the number of pupae in which the emergence of parasitoids occurred in the natural host $D$. saccharalis, and increased the longevity of females and the sex ratio of $T$. diatraeae. The progeny, duration of developmental cycle (egg to adult), width of head capsule of males and females and longevity of males of T. diatraeae were similar on both treatments. T. diatraeae can be reared in the factitious host $T$. molitor for three generations without compromising its reproductive performance, when subsequently reared in the natural host $D$. saccharalis.
\end{abstract}

KEY-WORDS: Parasitoids; pupae; biological quality.

\section{INTRODUÇÃO}

Os parasitoides são componentes importantes da fauna, devido ao seu papel no controle da população de outros insetos (Perioto et al. 2002). O parasitismo e o desenvolvimento (ciclo ovo-adulto) de himenópteros parasitoides de pupas de lepidópteros de importância agrícola ou florestal têm sido

\section{RESUMO}

A criação sucessiva de parasitoides em hospedeiros alternativos pode afetar sua qualidade biológica. Trichospilus diatraeae Cherian \& Margabandhu, 1942 (Hymenoptera: Eulophidae) tem sido estudado para o controle biológico da broca-da-cana [Diatraea saccharalis (Fabricius, 1794) (Lepidoptera: Crambidae)]. Objetivou-se avaliar se a criação de T. diatraeae, por três gerações, no hospedeiro alternativo Tenebrio molitor Linnaeus, 1758 (Coleoptera: Tenebrionidae), afeta seu desempenho reprodutivo, quando criado, posteriormente, em pupas do hospedeiro natural $D$. saccharalis. Dois grupos de T. diatraeae foram multiplicados, separadamente e por três gerações: um em pupas de T. molitor e outro em pupas de $D$. saccharalis. Na sequência, 20 pupas de $D$. saccharalis foram expostas, por 72 horas, ao parasitismo de fêmeas de T. diatraeae, criado, anteriormente, em pupas de T. molitor ou de $D$. saccharalis. A criação sucessiva de $T$. diatraeae no hospedeiro alternativo não afetou o número de pupas parasitadas e de pupas com emergência de parasitoides no hospedeiro natural $D$. saccharalis e aumentou a longevidade de fêmeas e a razão sexual de $T$. diatraeae. A progênie, duração do ciclo de desenvolvimento (ovo-adulto), largura da cápsula cefálica de machos e fêmeas e a longevidade de machos de $T$. diatraeae foram semelhantes entre os tratamentos. T. diatraeae pode ser criado no hospedeiro alternativo T. molitor, por três gerações, sem comprometer seu desempenho reprodutivo, ao ser criado, posteriormente, no hospedeiro natural D. saccharalis.

PALAVRAS-CHAVE: Parasitoides; pupas; qualidade biológica.

estudados ao longo dos anos (Bittencourt \& Berti Filho 2004, Pereira et al. 2009, Pereira et al. 2010b, Rodrigues et al. 2013). Entretanto, a dificuldade para a criação contínua de hospedeiros naturais de parasitoides e predadores, em laboratório, exige o uso de hospedeiros alternativos (Parra et al. 2002, Milward-de-Azevedo et al. 2004, Pereira et al. 2009, Pereira et al. 2010a, Zanuncio et al. 2008).

1. Trabalho recebido em jul./2013 e aceito para publicação em jun./2014 (nº registro: PAT 25410).

2. Universidade Federal da Grande Dourados (UFGD), Faculdade de Ciências Agrárias, Dourados, MS, Brasil.

E-mails: daniglaeser@yahoo.com.br, elileitevargas@gmail.com,nessacalado@hotmail.com.

3. Universidade Federal da Grande Dourados (UFGD), Faculdade de Ciências Biológicas e Ambientais, Dourados, MS, Brasil.

E-mails: fabriciofagundes@ufgd.edu.br, kellenfavero@yahoo.com.br. 
Os hospedeiros alternativos podem reduzir os custos de produção de criação massal de inimigos naturais (Ramalho \& Dias 2003, Dias et al. 2008, Pereira et al. 2010c). Contudo, a criação de parasitoides, por várias gerações, em um hospedeiro alternativo, pode afetar a preferência pelo hospedeiro natural, diminuindo a eficiência do controle biológico (Volpe et al. 2006).

Trichospilus diatraeae Cherian \& Margabandhu, 1942 (Hymenoptera: Eulophidae) é um endoparasitoide pupal e polífago, com potencial para o controle de Diatraea saccharalis (Fabricius, 1794) (Lepidoptera: Crambidae) (Paron \& Berti Filho 2000, Vargas et al. 2013), uma praga importante da cana-de-açúcar, com ampla distribuição, intensidade de ataque e danos econômicos (Portela et al. 2011). Este parasitoide pode ser criado em laboratório, em pupas do hospedeiro natural D. saccharalis e, também, em pupas do hospedeiro alternativo Tenebrio molitor Linnaeus, 1758 (Coleoptera: Tenebrionidae) (Favero et al. 2013). A utilização de T. molitor como hospedeiro alternativo, para a multiplicação de $T$. diatraeae, justifica-se pela facilidade de criação em laboratório (Favero et al. 2013) e pelo baixo custo de produção de pupas de T. molitor (Pires et al. 2009, Bortoli et al. 2011).

A adequabilidade de parasitoides polífagos a hospedeiros alternativos varia (Dias et al. 2010) e seus aspectos reprodutivos podem variar com o número de gerações em laboratório (Pratissoli et al. 2004, Dias et al. 2008). Além disto, a manutenção das características biológicas, visando ao controle de qualidade, é uma das etapas básicas de programas de criação de insetos em laboratório, para que os mesmos tenham características semelhantes àquelas de indivíduos encontrados em seu ambiente natural (Gonçalves et al. 2003)

Este trabalho objetivou avaliar o desempenho reprodutivo de $T$. diatraeae, em pupa de D. saccharalis, após três gerações no hospedeiro alternativo T. molitor.

\section{MATERIAL E MÉTODOS}

O trabalho foi desenvolvido no Laboratório de Entomologia e Controle Biológico (Lecobiol) da Faculdade de Ciências Agrárias da Universidade Federal da Grande Dourados (UFGD), em Dourados (MS), entre março e junho de 2010.

Pupas de $D$. saccharalis foram cedidas pela empresa BUG - Agentes Biológicos, para o estabe- lecimento da criação em laboratório. Foram mantidos 20 machos e 30 fêmeas adultos deste lepidóptero por gaiola de PVC $(10 \mathrm{~cm} \times 22 \mathrm{~cm})$, fechadas com tecido do tipo "voil" e elástico. Estas gaiolas foram revestidas com folhas de papel sulfite umedecido, onde as fêmeas depositaram as posturas. Após a eclosão, as lagartas de D. saccharalis foram transferidas para potes telados, contendo dieta artificial modificada de Hensley \& Hammond (1968), à base de farelo de soja, germe de trigo, vitaminas e sais minerais, onde permaneceram até a formação de pupas. As pupas foram recolhidas destes potes, sexadas e colocadas em gaiolas de PVC, até a emergência dos adultos (Parra 2007).

A criação do hospedeiro alternativo T. molitor iniciou-se com larvas provenientes de criação no Laboratório de Controle Biológico de Insetos da Universidade Federal de Viçosa (MG). As larvas deste Coleoptera foram mantidas em bandejas plásticas $(39,3 \mathrm{~cm} \times 59,5 \mathrm{~cm} \times 7,0 \mathrm{~cm})$ e alimentadas com farelo de trigo (97\%), levedo de cerveja (3\%) e chuchu (Zamperline \& Zanuncio 1992), até a fase de pupa, sendo utilizadas para a realização de experimentos com parasitoides ou acondicionadas em novas bandejas, até a fase adulta, para a manutenção da criação.

A população inicial de $T$. diatraeae foi proveniente de pupas de Thyrinteina arnobia (Stoll, 1782) (Lepidoptera: Geometridae) e criada, inicialmente, em pupas de Anticarsia gemmatalis Hübner, 1818 (Lepidoptera: Noctuidae), na Universidade Federal de Viçosa. Em agosto de 2007, este parasitoide passou a ser criado em pupas de T. molitor, no Lecobiol.

Os adultos de $T$. diatraeae foram mantidos em tubos de vidro $(14,0 \mathrm{~cm} \times 2,2 \mathrm{~cm})$ contendo uma gota de mel puro e fechados com algodão. Pupas de D. saccharalis, com 24 a 48 horas de idade, foram expostas ao parasitismo por 24 horas, para manutenção da criação deste parasitoide. Após este período, as pupas foram individualizadas em tubos de vidro e mantidas em câmara climatizada a $25 \pm 2^{\circ} \mathrm{C}, 70 \pm 10 \%$ de umidade relativa e fotofase de 14 horas, até a emergência de adultos (Paron \& Berti Filho 2000, Favero et al. 2013).

O parasitoide $T$. diatraeae foi criado, por três gerações, em pupas de Ephestia kuehniella Zeller, 1879 (Lepidoptera: Pyralidae) (hospedeiro neutro, para reduzir a possibilidade de condicionamento pré-imaginal ao hospedeiro de criação), para avaliar se a criação de $T$. diatraeae em pupas do coleóptero T. molitor, por três gerações (número de gerações 
definido em função das atividades realizadas no Lecobiol, alternando entre dois hospedeiros), afeta seu desempenho reprodutivo sobre a pupa do lepidóptero D. saccharalis.

Dois grupos de $T$. diatraeae foram multiplicados, separadamente, por três gerações: um no hospedeiro alternativo T. molitor e outro no hospedeiro natural $D$. saccharalis. Após esta sequência de criação, 20 pupas fêmeas de $D$. saccharalis foram pesadas, visando à padronização (peso médio de $0,167 \pm 0,01 \mathrm{~g}$ ), e individualizadas em tubos de vidro $(14,0 \mathrm{~cm} \mathrm{x}$ $2,2 \mathrm{~cm}$ ), onde permaneceram expostas por 72 horas ao parasitismo de sete fêmeas de T. diatraeae com 24 horas de idade, criadas, anteriormente, em pupas de T. molitor ou de D. saccharalis. Após este período, as fêmeas de $T$. diatraeae foram retiradas e as pupas permaneceram individualizadas em tubos de vidro e mantidas em sala climatizada, com temperatura de $25 \pm 2^{\circ} \mathrm{C}$, umidade relativa de $70 \pm 10 \%$ e fotofase de 14 horas, até a emergência de adultos de T. diatraeae (Favero et al. 2013). Ressalta-se que foram selecionadas, também, 20 pupas que não foram expostas ao parasitismo, com o objetivo de calcular a mortalidade natural do hospedeiro, caso necessário (Abbot 1925).

O delineamento experimental foi o inteiramente casualizado, com dois tratamentos representados por $T$. diatraeae, após ser criado em cada hospedeiro (T. molitor ou D. saccharalis), totalizando 20 repetições.

As características biológicas avaliadas foram o número de pupas parasitadas (pupas parasitadas são mais escuras e, geralmente, contêm imaturos em seu interior); número de pupas com emergência de T. diatraeae; número de parasitoides emergidos por pupa de $D$. saccharalis (progênie); duração do ciclo de desenvolvimento (ovo-adulto); longevidade média em dias (20 fềmeas e 10 machos de T. diatraeae foram selecionados ao acaso, por tratamento, no dia de sua emergência, e individualizados em tubos de vidro contendo uma gota de mel puro, da emergência até a morte); largura da cápsula cefálica do adulto (15 fêmeas e 15 machos de $T$. diatraeae foram selecionados ao acaso, visando a medir a largura da cápsula cefálica em ocular micrométrica); e razão sexual ( $\mathrm{RS}=$ número de fêmeas/ número de adultos) de T. diatraeae. O sexo dos parasitoides foi determinado de acordo com características morfológicas da antena e do abdome (Cherian \& Margabandhu 1942).

Os valores para número de pupas parasitadas ou com emergência de $T$. diatraeae foram subme- tidos à análise de modelos lineares generalizados com distribuição binomial $(\mathrm{p} \leq 0,05)$, com o auxílio do R Statistical System (Ihaka \& Gentleman 1996). Esta análise foi realizada com os dados originais não-paramétricos, representados por 0 (pupas não parasitadas e pupas sem emergência) e 1 (pupas parasitadas e pupas com emergência), gerando um teste de $\chi^{2}$. Os dados referentes à duração do ciclo de desenvolvimento, número de parasitoides emergidos por pupa de $D$. saccharalis (progênie), razão sexual, longevidade e largura da cápsula cefálica de fêmeas e machos de $T$. diatraeae foram submetidos à análise de variância, pelo teste $\mathrm{F}$, a 5\%.

\section{RESULTADOS E DISCUSSÃO}

A criação sucessiva de $T$. diatraeae, por três gerações, no hospedeiro alternativo T. molitor, e posterior criação no hospedeiro natural $D$. saccharalis, não afetou o número de pupas parasitadas, pois todas as pupas foram parasitadas, assim como não afetou o número de pupas com emergência de $T$. diatraeae $\left(\chi^{2}=33,019 ; p>0,05\right)$ (Figura 1).

A progênie, duração do ciclo de desenvolvimento, longevidade de machos e largura da cápsula cefálica de fêmeas e de machos de $T$. diatraeae emergidos de pupas de $D$. saccharalis apresentaram

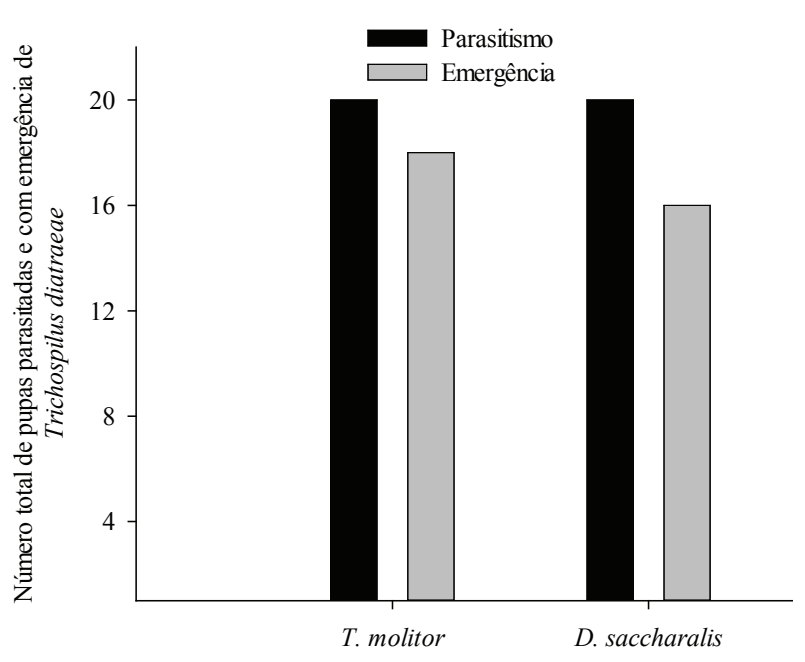

Figura 1. Número total de pupas de Diatraea saccharalis (Lepidoptera: Crambidae) parasitadas e de pupas parasitadas com emergência de Trichospilus diatraeae (Hymenoptera: Eulophidae), após este parasitoide ter sido criado, por três gerações, em pupas de Tenebrio molitor (Coleoptera: Tenebrionidae) ou D. saccharalis. Temperatura de $25 \pm 2^{\circ} \mathrm{C}$, umidade relativa de $70 \pm 10 \%$ e fotofase de 14 horas (Dourados, MS, 2010). 
valores semelhantes $(p>0,05)$. A longevidade de fêmeas $(\mathrm{F}=13,302 ; \mathrm{p}=0,0008)$ e a razão sexual de T. diatraeae $(\mathrm{F}=4,500 ; \mathrm{p}=0,0417)$ foram maiores em pupas de $D$. saccharalis parasitadas por fêmeas deste parasitoide provenientes de pupas de $T$. molitor (Tabela 1).

As taxas de parasitismo e de emergência, progênie, ciclo de desenvolvimento, longevidade de machos e largura da cápsula cefálica de fêmeas e machos de T. diatraeae, emergidos de pupas do hospedeiro natural $D$. saccharalis, foram semelhantes, após três gerações em pupas do hospedeiro alternativo T. molitor, não tendo, assim, comprometido a qualidade biológica deste parasitoide. A qualidade de um parasitoide é determinada por um conjunto de características biológicas (Roitberg et al. 2001, Prezotti et al. 2002), incluindo as avaliadas neste trabalho.

Os resultados obtidos neste trabalho são semelhantes aos observados por Pereira et al. (2010b), em que os aspectos biológicos (parasitismo, emergência, progênie por pupa, tamanho do corpo, largura da cápsula cefálica de fêmeas e machos e longevidade de machos) de Palmistichus elaeisis Delvare \& LaSalle, 1993 (Hymenoptera: Eulophidae), em pupas do hospedeiro natural Thyrinteina arnobia (Stoll, 1782) (Lepidoptera: Geometridae), não foram comprometidos, após o mesmo ter sido criado, por seis gerações, em pupas dos hospedeiros alternativos Anticarsia gemmatalis Hubner, 1818 (Lepidoptera: Noctuidae) ou Bombyx mori Linnaeus, 1758 (Lepidoptera: Bombycidae) (Pereira et al. 2010b).

A criação sucessiva de $T$. diatraeae, por três gerações, em pupas de T. molitor, e posterior criação no hospedeiro natural $D$. saccharalis, aumentou a longevidade de fêmeas e a razão sexual deste para- sitoide. O uso de hospedeiros alternativos, muitas vezes, pode contribuir para a melhoria das criações de parasitoides em laboratório, como, também, foi constatado para espécies de Trichogramma (Hymenoptera: Trichogrammatidae), devido à redução de custos e aumento da eficiência destes parasitoides, durante o processo de produção massal (Dias et al. 2008).

T. diatraeae deve ser criado em, pelo menos, dois hospedeiros, em laboratório. Além disto, indivíduos de T. diatraeae coletados no campo devem ser, esporadicamente, introduzidos nas criações, por terem maior variabilidade genética e poderem contribuir para a manutenção da qualidade biológica deste parasitoide. Isto é necessário, pois a criação de parasitoides, por várias gerações, em um único hospedeiro (alternativo ou natural), pode reduzir suas qualidades biológicas, sendo importante alternar as criações em, pelo menos, dois hospedeiros, para minimizar o efeito da possível diminuição da variabilidade genética destes inimigos naturais (Bartlett 1985, Bueno 2009).

A criação contínua de inimigos naturais, em laboratório, pode aumentar a produção de indivíduos homozigóticos, em relação às populações no campo, os quais podem apresentar características indesejáveis. Entretanto, a obtenção de grandes populações destes insetos pode contribuir para a manutenção da variabilidade genética e para o controle de qualidade das criações (Bartlett 1985, Gonçalves et al. 2003).

O desempenho reprodutivo e as características biológicas de $T$. diatraeae não foram afetados, negativamente, pela criação, após três gerações, no hospedeiro alternativo T. molitor, e posterior criação no hospedeiro natural $D$. saccharalis. Isto é relevante

Tabela 1. Características biológicas (média \pm erro padrão) de Trichospilus diatraeae (Hymenoptera: Eulophidae), em pupas de Diatraea saccharalis (Lepidoptera: Crambidae), após sua criação, por três gerações, em pupas de Tenebrio molitor (Coleoptera:Tenebrionidae) ou $D$. saccharalis, em laboratório, a $25 \pm 2^{\circ} \mathrm{C}, 70 \pm 10 \%$ de umidade relativa e fotofase de 12 horas (Dourados, MS, 2010).

\begin{tabular}{lcccc}
\hline \multicolumn{1}{c}{ Característica biológica } & Tenebrio molitor & $\mathrm{n}$ & D. saccharalis & $\mathrm{n}$ \\
\hline Ciclo de desenvolvimento (dias) & $17,40 \pm 0,76 \mathrm{a}$ & 18 & $16,33 \pm 0,69 \mathrm{a}$ & 16 \\
Progênie/pupa & $401,20 \pm 58,12 \mathrm{a}$ & 18 & $380,44 \pm 38,04 \mathrm{a}$ & 16 \\
Cápsula cefálica do macho (mm) & $0,36 \pm 0,01 \mathrm{a}$ & 15 & $0,37 \pm 0,01 \mathrm{a}$ & 15 \\
Cápsula cefálica da fêmea (mm) & $0,43 \pm 0,01 \mathrm{a}$ & 15 & $0,43 \pm 0,01 \mathrm{a}$ & 15 \\
Longevidade da fêmea (dias) & $18,65 \pm 0,52 \mathrm{a}$ & 20 & $15,35 \pm 0,74 \mathrm{~b}$ & 20 \\
Longevidade do macho (dias) & $17,40 \pm 0,31 \mathrm{a}$ & 10 & $15,50 \pm 1,26 \mathrm{a}$ & 10 \\
Razão sexual & $0,89 \pm 0,02 \mathrm{a}$ & 18 & $0,83 \pm 0,02 \mathrm{~b}$ & 16 \\
\hline
\end{tabular}

Médias seguidas de mesma letra minúscula, na linha, não diferem entre si, pelo teste $\mathrm{F}$, a 5\%. n: número de pupas com emergência, para avaliação da duração do ciclo de vida, progênie e razão sexual, e número de indivíduos, para avaliação da longevidade e largura da cápsula cefálica. 
para a multiplicação e manutenção de $T$. diatraeae, em laboratório, pois a criação de T. molitor é de baixo custo e pode suprir a ausência temporária de pupas de $D$. saccharalis.

\section{CONCLUSÃO}

A criação de T. diatraeae, por três gerações, no hospedeiro alternativo T. molitor, não comprometeu seu desempenho reprodutivo, ao ser criado, posteriormente, no hospedeiro natural $D$. saccharalis, e favoreceu a melhoria de características biológicas, como longevidade de fêmeas e razão sexual.

\section{REFERENNCIAS}

ABBOTT, W. S. A method of computing the effectiveness of an insecticide. Journal of Economic Entomology, Lanham, v. 18, n. 2, p. 265-267, 1925.

BARTLETT, A. C. Guidelines for genetic diversity in laboratory colony establishment and maintenance. In: SINGH, P.; MOORE, R. F. (Eds.). Handbook of insect rearing. Amsterdam: Elsevier, 1985. p. 7-17.

BITTENCOURT, M. A. L.; BERTI FILHO, E. Desenvolvimento dos estágios imaturos de Palmistichus elaeisis Delvare \& LaSalle (Hymenoptera, Eulophidae) em pupas de Lepidoptera. Revista Brasileira de Entomologia, Piracicaba, v. 48, n. 4, p. 65-68, 2004.

BORTOLI, S. A. de et al. Comparative biology and production costs of Podisus nigrispinus (Hemiptera: Pentatomidae) when fed different types of prey. Biological Control, Amsterdam, v. 58, n. 2, p. 127-132, 2011.

BUENO, V. H. P. Controle biológico de pragas: produção massal e controle de qualidade. Lavras: UFLa, 2009.

CHERIAN, M. C.; MARGABANDHU, V. A new species of Trichospilus (Hymenoptera: Chalcidoidea) from south India. Indian Journal of Entomology, New Delhi, v. 4, n. 2, p. 101-102, 1942.

DIAS, N. da S.; PARRA, J. R. P.; DIAS, C. T. dos S. Tabela de vida de fertilidade de três espécies neotropicais de Trichogrammatidae em ovos de hospedeiros alternativos como critério de seleção hospedeira. Revista Brasileira de Entomologia, São Paulo, v. 54, n. 1, p. 120-124, 2010.

DIAS, N. da S.; PARRA, J. R. P.; LIMA, T. C. da C. Seleção de hospedeiro alternativo para três espécies de tricogramatídeos neotropicais. Pesquisa Agropecuária Brasileira, Brasília, DF, v. 43, n. 11, p. 1467-1473, 2008.

FAVERO, K. et al. Biological characteristics of Trichospilus diatraeae (Hymenoptera: Eulophidae) are influenced by the number of females exposed per pupa of Tenebrio molitor (Coleoptera: Tenebrionidae). Florida Entomologist, Gainesville, v. 96, n. 2, p. 583-589, 2013.

GONÇALVES, J. R. et al. Avaliação da qualidade de Trichogramma pretiosum (Hymenoptera: Trichogrammatidae) em ovos de Sitotroga cerealella (Lepidoptera: Gelechiidae). Acta Scientiarum Agronomy, Maringá, v. 25, n. 2, p. 485-489, 2003.

HENSLEY, S. D.; HAMMOND, A. H. Laboratory techniques for rearing the sugar cane borer on an artificial diet. Journal of Economic Entomology, Lanham, v. 61, n. 6, p. 1742-1743, 1968.

IHAKA, R.; GENTLEMAN, R. R: a language for data analysis and graphics. Journal of Computational and Graphical Statistics, Montreal, v. 5, n. 3, p. 299-314, 1996.

MILWARD-DE-AZEVEDO, E. M. V. et al. Desempenho reprodutivo de Nasonia vitripennis Walker (Hymenoptera: Pteromalidae) em pupas crioconservadas de Chrysomia megacephala Fabricius (Diptera: Calliphoridae): avaliação preliminar. Ciência Rural, Santa Maria, v. 34, n. 1, p. 207 211, 2004.

PARRA, J. R. P. Técnicas de criação de insetos para programas de controle biológico. 6. ed. Piracicaba: ESALQ/FEALQ, 2007.

PARRA, J. R. P. et al. Controle biológico no Brasil: parasitoides e predadores. São Paulo: Manole, 2002.

PARON, M. R.; BERTI FILHO, E. Capacidade reprodutiva de Trichospilus diatraeae (Hymenoptera: Eulophidae) em pupas de diferentes hospedeiros (Lepidoptera). Scientia Agricola, Piracicaba, v. 57, n. 2, p. 355-358, 2000.

PEREIRA, F. F. et al. Progênie de Palmistichus elaeisis Delvare \& LaSalle (Hymenoptera: Eulophidae) parasitando pupas de Bombyx mori L. (Lepidoptera: Bombycidae) de diferentes idades. Neotropical Entomology, Londrina, v. 38, n. 5, p. 660-664, 2009.

PEREIRA, F. F. et al. Parasitismo de Palmistichus elaeisis (Hymenoptera: Eulophidae) em hospedeiro alternativo sobre plantas de eucalipto em semicampo. Revista Ciência Agronômica, Fortaleza, v. 41, n. 4, p. 715-720, 2010a.

PEREIRA, F. F. et al. Reproductive biology of Palmistichus elaeisis (Hymenoptera: Eulophidae) with alternative and natural hosts. Zoologia, Curitiba, v. 27, n. 6, p. 887-891, 2010 b.

PEREIRA, F. F. et al. The density of females of Palmistichus elaeisis Delvare and LaSalle (Hymenoptera: Eulophidae) affects their reproductive performance on pupae of Bombyx mori L. (Lepidoptera: Bombycidae). Anais da Academia Brasileira de Ciências, Rio de Janeiro, v. 82, n. 2, p. 323-331, 2010c. 
PERIOTO, N. W. et al. Himenópteros parasitoides (Insecta, Hymenoptera) coletados em cultura de algodão (Gossypium hirsutum L.) (Malvaceae), no município de Ribeirão Preto, SP, Brasil. Revista Brasileira de Entomologia, São Paulo, v. 46, n. 2, p. 165-168, 2002.

PIRES, E. M. et al. Desenvolvimento, reprodução e performance predatória do percevejo zoofitófago Podisus distinctus (Hemiptera: Pentatomidae) alimentado com larvas de Musca domestica (Diptera: Muscidae) imobilizadas ou soltas. Revista Brasileira de Biociências, Porto Alegre, v. 7, n. 3, p. 280-284, 2009.

PORTELA, G. L. F. et al. Infestação de Diatraea spp. em diferentes variedades de cana-de-açúcar em União - PI. Revista Caatinga, Mossoró, v. 24, n. 1, p. 149-152, 2011.

PRATISSOLI, D. et al. Fertility life table of Trichogramma pretiosum and Trichogramma acacioi on eggs of Anagasta kuehniella at different temperatures. Pesquisa Agropecuária Brasileira, Brasília, DF, v. 39, n. 2, p. 193196, 2004.

PREZOTTI, L. et al. Flight test as evaluation criterion for the quality of Trichogramma pretiosum Riley (Hymenoptera: Trichogrammatidae): adaptation of the methodology. Neotropical Entomology, Londrina, v. 31, n. 3, p. 411-417, 2002.

RAMALHO, F. S.; DIAS, J. M. Efeitos de hospedeiros alternativos na biologia de Catolaccus grandis (Burks) (Hymenoptera: Pteromalidae), parasitoide de Anthonomus grandis Boheman (Coleoptera: Curculionidae). Neotropical Entomology, Londrina, v. 32, n. 2, p. 305-310, 2003.
RODRIGUES, M. A. T. et al. Thermal requirements and generation estimates of Trichospilus diatraeae (Hymenoptera: Eulophidae) in sugarcane producing regions of Brazil. The Florida Entomologist, Lutz, v. 96, n. 1, p. 154-159, 2013.

ROITBERG, B. D.; BOIVIN, G.; VET, L. E. M. Fitness, parasitoids, and biological control: an opinion. Canadian Entomologyst, Quebec, v. 133, n. 3, p. 429-438, 2001.

VARGAS, E. L. et al. Searching and parasitism of Diatraea saccharalis (Lepidoptera: Crambidae) by Trichospilus diatraeae (Hymenoptera: Eulophidae). Acta Biologica Colombiana, Bogotá, v. 18, n. 2, p. 259-264, 2013.

VOLPE, H. X. L. et al. Avaliação de características biológicas de Trichogramma pretiosum Riley (Hymenoptera: Trichogrammatidae) criado em três hospedeiros. Arquivos do Instituto Biológico, São Paulo, v. 73, n. 3, p. 311-315, 2006.

ZAMPERLINE, B.; ZANUNCIO, J. C.; Influência da alimentação de Tenebrio molitor L. 1758 (Coleoptera: Tenebrionidae) no desenvolvimento ninfal de Podisus connexivus Bergroth, 1891 (Hemiptera: Pentatomidae). Revista Árvore, Viçosa, v. 16, n. 2, p. 224-230, 1992.

ZANUNCIO, J. C. et al. Tenebrio molitor Linnaeus (Coleoptera: Tenebrionidae): a new alternative host to rear the pupae parasitoid Palmistichus elaeisis Delvare \& LaSalle (Hymenoptera: Eulophidae). The Coleopterists Bulletin, Washington, D.C., v. 62, n. 1, p. 64-66, 2008. 